\title{
Measurement method of the anterior chamber volume by image analysis
}

\author{
TAKEHISA KONDO, ${ }^{1}$ MASAO MIURA,' AND MASATSUGU IMAMICHI ${ }^{2}$ \\ From the 'Department of Ophthalmology, Kobe General Hospital, and ${ }^{2}$ San Contact Lens Co. Ltd, Japan
}

SUMMARY A new computerised method of measurement of the anterior chamber in the human eye is described. Photographs of the anterior chamber were taken with a Zeiss slit-lamp 75-SL and converted from an optical image into a true image by computer with a digitiser. We obtained data on both the anterior chamber volume and also the anterior chamber depth, peripheral anterior chamber volume, the diameter of the anterior chamber, and the degree of the iridocorneal angle. These data were simultaneously displayed several seconds after in-put by the digitiser. In mensuration by digitiser on three occasions from a single photograph of a given eye the mean deviation rate was about $0.7 \%$, and the mean deviation rate of 10 successive exposures of one eye was $3 \cdot 5 \%$. By this image analysis technique an accurate profile of the anterior chamber is displayed of the pupil margin, iris-root insertion, and central anteroposterior pupillary axis. These data help us to understand the mechanism of primary angle-closure glaucoma.

Methods of measuring the anterior chamber volume have been described. But they have been somewhat difficult to apply in clinical examination and have not been sufficiently perfected to be useful in an outpatient setting. Recently Johnson et al.' reported on a new simple method for measuring the anterior chamber volume, in which they used an unaltered, commercially available slit-lamp camera system. In this method the anterior chamber volume is calculated from the anterior chamber scale obtained from a plastic model eye together with a transparent optically distorted grid overlay.

We have developed another convenient method of measuring the anterior chamber volume. It is a new computerised method for converting the optically distorted image into a true image and for calculating the volume from the true dimensions. We can furthermore obtain a true profile of the anterior chamber such as pupil margin and iris root insertion. The true profile provides data for comparing normal eyes with glaucomatous eyes.

The purpose of this report is, first, to describe our new simplified optical method for measuring the anterior chamber volume and for providing a diagram of the anterior chamber profile by means of a computer with a digitiser. Secondly, we discuss the accuracy of the values obtained by this method.

Correspondence to Dr T Kondo, Department of Ophthalmology, Kobe General Hospital, 4-6, Minatojima-Nakamachi, Chuo-ku, Kobe 650, Japan.

\section{Materials and methods}

The profile of the anterior eye segment was photographed with Zeiss slit-lamp 75-SL. The vertical slitbeam was projected along the visual axis of the eye, and the camera was locked at an angle of $45^{\circ}$ to the left for the slit-lamp with the use of the longest slit. Shutter speed was $1 / 60$ second, photoadapter 14 , flash impulse 4 , zoom factor $1 \cdot 0$, illumination of the background $25 \%$, width of the slit-beam $0 \cdot 1$, film Kodak (ASA 400).

The colour photographs, developed and enlarged, were analysed by a digitiser, and the optical image of the anterior eye segment was converted into the true image by computer. A given point $\left(x_{i}, y_{i}\right)$ was converted into the point $\left(x_{r}, y_{r}\right)$ in the true image according to the following equations:

$$
\begin{aligned}
& r_{i}=\sqrt{x^{2}+y^{2} i} \\
& \zeta_{i}=\arctan \left(y_{i} / x_{i}\right) \\
& \vartheta=\arcsin \left(r_{i} / r_{i}\right) \\
& \vartheta^{\prime}=\arcsin (\sin \vartheta / n) \\
& r=\frac{r_{k} \cdot \sin \vartheta-r_{k} \cos \vartheta \cdot \tan \left(\vartheta-\vartheta^{\prime}\right)}{1-\sin \zeta_{i} \cdot \tan \left(\vartheta-\vartheta^{\prime}\right)} \\
& Z=r \sin \zeta_{i} \\
& X=r \cos \zeta_{i} \\
& Y=r \sin \zeta_{i} \\
& X_{r}=x \\
& Y_{r}=\sqrt{y^{2}+Z^{2}}
\end{aligned}
$$

where

$\mathrm{x}_{\mathrm{i}}$ is the distance from the axis in the optical image, $y_{i}$ is the height at the $x_{i}$ point in the anterior chamber 
in the optical image, $r_{i}$ is the distance from the centre of the true corneal radius to the point $\left(x_{i}, y_{i}\right)$ in the polar co-ordinate, and $r$ is the true distance of $r_{i}$, and $r_{k}$ is the true corneal radius, $n$ is the refractive power of aqueous humor, $\mathrm{x}_{\mathrm{r}}$ is the distance from the optic axis in the true image, $y_{r}$ is the height at the $x_{r}$ point in the true image, the $x$ axis is set in the vertical plane, the $\mathrm{y}$ axis is set in the horizontal plane, the $\mathrm{z}$ axis is set in the rectangular plane to the $x-y$ plane, $x_{r}$ is set in the vertical axis and $y_{r}$ is set in the optic axis in the true image, and $r$ is the true distance of $r_{i}, \zeta_{i}$ is the angle in the polar co-ordinate between the $x$ axis and the line from the point $\left(x_{i}, y_{i}\right)$ to the centre of the corneal radius, $\vartheta$ is the angle of incidence, and $\vartheta^{\prime}$ is the angle of refraction.

The true depth of the anterior chamber at intervals of about $0.5 \mathrm{~mm}$ from the optic axis of the eye to the iridocorneal angle is read directly from the photograph by a digitiser. According to the method of estimation of the anterior chamber volume described by Johnson $e t$ al. ' the anterior chamber volume was assumed to be equal to the volume of revolution of its central sagittal section rotated $180^{\circ}$ about the optic axis. The volume of such a solid can be approximated from the sums of the volumes of a series of concentric right circular cylinders, the heights of which are equal to the anterior chamber depths at each of a series of intervals. The upper half and the lower half of the anterior chamber were estimated separately in the present study. Each anterior chamber depth measurement multiplied by computer gives the volume of one of a series of concentric half cylindrical sleeves, the sums of which equal half an anterior chamber volume. The anterior chamber volume consists of the sums of each half of the upper part and the lower part (Fig. 1). These steps can be quickly carried out with the aid of a computer, and, in

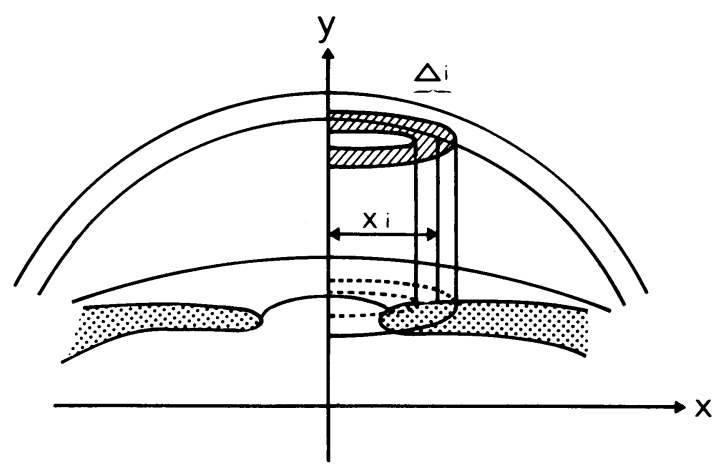

Fig. 1 The upper half and the lower half of the anterior chamber were estimated separately. The anterior chamber volume consists of the sums of each half of the upper part and the lower part, consisting of a series of concentric half cylindrical sleeves. ${ }^{7}$

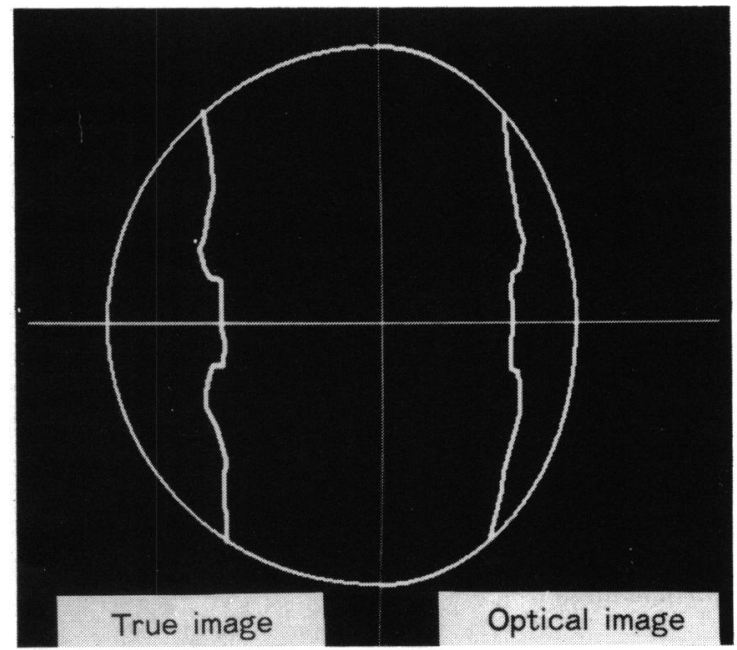

Fig. 2 The true profile of the anterior chamber was displayed by converting from the optical image to the true image by the computer after input with a digitiser. The true image is displayed on the left side, the optical image on the right.

addition to the data of the anterior chamber volume, several kinds of data such as the peripheral anterior chamber volume, the anterior chamber depth, the diameter of the anterior chamber, the degree of the iridocorneal angle, and the true profile of the anterior chamber are displayed within several seconds (Fig. 2).

The magnification rate was evaluated by a micrometer disc (Zeiss) fixed on the pointer of the fixation

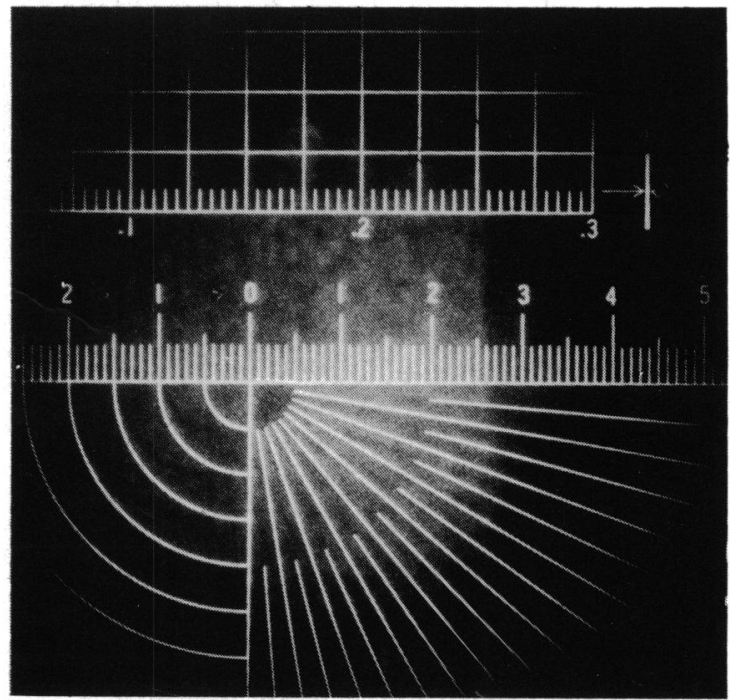

Fig. 3 The picture of a micrometer disc (Zeiss). The unit of length is $0.01 \mathrm{~mm}$. 
light. The micrometer disc (units of $0.01 \mathrm{~mm}$ ) was photographed on to the film for the anterior segment of the eye (Fig. 3).

We used a PC-9801F (Nihon Electronic Co.) computer and a Gradimate-SQ-3000 (Oskon Denshi) digitiser for our study.

Our method has been developed from the following assumptions. The corneal surface is spherical with a radius of $r_{k}$. The refractive indices of the cornea and the aqueous humour are equal. The thickness of the cornea is $0.6 \mathrm{~mm}$ over the entire surface of the cornea. The interval between $x_{i}$ and $x_{i+1}$ on the iris surface or the lens surface is linear. And the true image of the iridocorneal angle is estimated as the part surrounding both the corneal curvature and the straight line along the iris surface beside the iridocorneal angle.

\section{Results}

The consistency of the mensuration procedure was examined by making three estimates of the volume from a single photograph on separate occasions. The results are shown in Table 1.

The consistency of the photographic technique and mensuration procedure with a digitiser was examined by taking 10 exposures of one eye and reading the photograph with a digitiser. The results are shown in Table 2.

\section{Discussion}

Several methods for measuring the anterior chamber volume from a slit-image photograph have been described.$^{1-7}$ Each method preceding that of Johnson et al. ${ }^{1}$ requires special equipment, with slit-lamp modification. Johnson et al. ' recently reported on a simple optical method for measuring the anterior chamber volume by means of an unmodified, commercially available slit-lamp system and transparent anterior chamber scales. We have developed a new method of measuring the anterior chamber volume. It is an image analysis method for conversion of the optically distorted image into a true image and for calculation of the volume from the dimensions by computer with a digitiser. Both steps are simultaneously carried out by a commercially available computer with a digitiser, and the entire mensuration procedure takes less than 10 seconds. Our method requires neither special equipment nor modification of a standard slit-lamp system. From the distorted image given by a slit-lamp photograph a correctly dimensioned outline of the cross section of the anterior chamber is derived, point by point, by means of a digitiser. This method allows the anterior chamber volume to be derived rapidly from the true
Table 1 Volume of anterior chamber of a subject by mensuration on three occasions from a single photograph of the eye

\begin{tabular}{llll}
\hline Experiment & 1 & 2 & 3 \\
\hline Volume $(\mu \mathrm{l})$ & 195.6 & 198.8 & 196.8 \\
\hline
\end{tabular}

Mean deviation $=1 \cdot 32 \mu \mathrm{l}(0 \cdot 7 \%)$.

Table 2 Volume of anterior chamber of a subject obtained from 10 successive photographs

\begin{tabular}{ll}
\hline Experiment & Volume $(\mu l)$ \\
\hline 1 & $139 \cdot 6$ \\
2 & $138 \cdot 7$ \\
3 & $138 \cdot 1$ \\
4 & $151 \cdot 1$ \\
5 & $151 \cdot 4$ \\
6 & $140 \cdot 0$ \\
7 & $146 \cdot 0$ \\
8 & $139 \cdot 3$ \\
9 & $149 \cdot 7$ \\
10 & $145 \cdot 7$ \\
\hline
\end{tabular}

Mean deviation $=5 \cdot 04 \mu \mathrm{l}(3 \cdot 5 \%)$.

image of the anterior chamber, and it also allows the peripheral anterior chamber volume, the anterior chamber depth, the peripheral anterior chamber depth, the diameter of the anterior chamber, and the degree of the iridocorneal angle to be derived at the same time. Furthermore this method provides an estimate of the profile of the anterior chamber-that is, the relative positions of pupil margin, lens surface, iris root insertion, and anteroposterior lens axis.

Although $\mathrm{Heim}^{2}$ pointed out that it is generally impossible to obtain a view of both angles of the anterior chamber together with vertical slit-beam, fortunately the Zeiss slit-lamp 75-SL has a long enough slit-beam to obtain a view of both angles even with the vertical slit-beam, unlike other slit-lamps. This enabled us to photograph the anterior chamber with the vertical slit-beam. In addition the vertical slit-beam has the advantage of making the measurement accurately, because the width of the anterior chamber angle does not seem to be equal in different sections of the chamber, while there is a great difference between upper half anterior chamber volume and lower half. We recommend the evaluation of data of the anterior chamber volume using the sums of the values of both the upper half and the lower half.

Nagataki et al. ${ }^{8}$ recently reported on a modified method for calculating the anterior chamber volume. The advantage of this method is that the true anterior chamber dimensions can be measured in an eye of any corneal curvature. By using a digitiser we can also apply this method of measurement to the cases of 


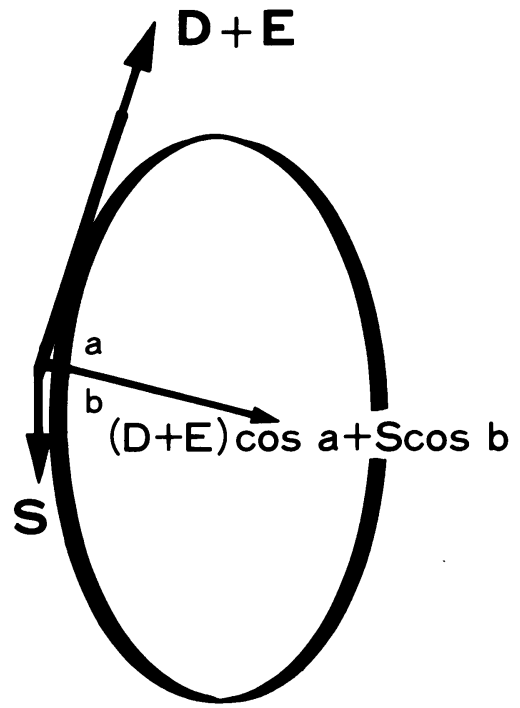

Fig. 4 Pupil blocking force (F) consists of $(\mathrm{D}+\mathrm{E}) \cos \mathrm{a}+\mathrm{S} \cos \mathrm{b}$. where $\mathrm{D}$ is pupil dilating force. $\mathrm{E}$ is iris-stretching force. $\mathrm{S}$ is sphincter contracting force.

radius of the corneal curvature, without the transparent anterior chamber scales reported by Johnson et al. ${ }^{\prime}$

The most important point for maintaining the accuracy of measurement in the photogrammetric method is the precise evaluation of the magnification ratio of the pictures. We fixed a micrometer disc on the fixation pointer to ensure that a constant distance was maintained between the camera and the top of the cornea. This method is particularly accurate for estimating the magnification ratio, because the micrometer disc is photographed on to the film of the anterior chamber. Each photograph was enlarged 5-6 times in the present study. In using a large magnification we reduce the mensuration errors of the digitiser. Using one photograph of a given specimen we tested the mensuration by digitiser three times, and obtained a mean deviation of about $\mathbf{0 . 7 \%}$. This suggests that the mensuration procedure by digitiser is not a substantial source of error.

Another important point during the taking of photographs is the centring of the slit-beam against the cornea. Small errors in alignment of the centre of the slit-beam with the optic axis of the eye are bound to occur. ${ }^{39}$ In our studies the mean deviation of $\mathbf{1 0}$ successive exposures of one eye was $3 \cdot 5 \%$. Successively exposed pictures, except for those with misalignment of the centre of the slit-beam, should be selected for the estimation of the anterior chamber volume.

From the true profile of the anterior chamber

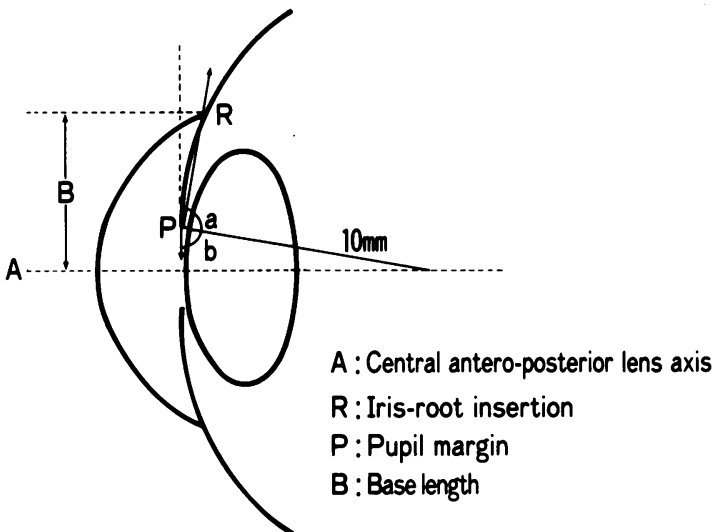

Fig. 5 With a digitiser base length, pupil margin, iris-root insertion, central anteroposterior pupillary axis, angle $a$, and angle $b$ are precisely estimated from the true image.

drawn by computer with a digitiser we can evaluate the direction of the vectors, such as pupil dilating force (D), iris-stretching force (E), and sphincter contracting force (S) as described by Mapstone. "' The calculation of these vectors will result in the estimation of the value of the pupil blocking force $(F)$ by the following equation:

$$
F=(D+E) \cos a+S \cos b
$$

where $a$ is the angle consisting of both the vector $(D+E)$ and the line from the pupil margin to the centre of the radius of the lens surface, and $b$ is the angle consisting of both the vector $S$ and the line from the pupil margin to the centre of the radius of the lens surface (Fig. 4). These angles (a,b) are estimated from the true image of the anterior chamber drawn by digitiser (Fig. 5). Thus we can calculate the pupil blocking force in each eye.

This new image analysis method of measuring the anterior chamber volume is convenient for both clinical examination and basic research. The precise evaluation of the anterior chamber volume provides beneficial data for physiopathological research into the dynamics of the aqueous humour, and the accurate profile of the anterior chamber provides important data for an analysis of the mechanism of glaucomatous attacks.

\section{References}

1 Johnson SB, Passmore JA, Brubaker RF. The fluorescein distribution volume of the anterior chamber. Invest Ophthalmol Vis Sci 1977; 16: 633-6.

2 Heim M. Phtographische Bestimmung der Teife und des Volumens der menschlichen Vorderkammer. Ophthalmologica 1941; 102: 13-220.

3 Jones RF, Maurice DM. A simple photographic method of measuring the volume of the anterior chamber. Exp Eye Res $1963 ; 2$ : 233-6. 
4 Brown N. Quantitative slit-image photography of the anterior chamber. Trans Ophthalmol Soc UK 1973; 93: 277-86.

5 Tomlinson A, Leighton DA. Ocular dimensions and the heredity of angle-closure glaucoma. Br J Ophthalmol 1973; 57: 475-86.

6 Fontana ST, Brubaker RF. Volume and depth of the anterior chamber in the normal aging human eye. Arch Ophthalmol 1980; 98: $1803-8$.

7 Kondo T, Miura M, Imamichi M. Computerized photogrammetric method of measurement of the anterior chamber volume. Nippon Ganka Gakkai Zasshi 1985; 89: 850-5
8 Nagataki S, Lee DA, Iri M. A simple geometrical method of measuring anterior chamber dimensions. Invest Ophthalmol Vis Sci 1984; 25: 1192-7.

9 Johnson SB, Coakes RL, Brubaker RF. A simple photogrammetric method of measuring anterior chamber volume. Am J Ophthalmol 1978; 85: 469-74.

10 Mapstone R. Mechanics of pupil block. Br J Ophthalmol 1968; 52: 19-25.

Accepted for publication 2 January 1986. 